\title{
BMJ Open Psychotherapy for depression in children and adolescents: study protocol for a systematic review and network meta-analysis
}

\author{
Bin Qin, ${ }^{1}$ Xinyu Zhou, ${ }^{1}$ Kurt D Michael, ${ }^{2}$ Yiyun Liu, ${ }^{1}$ Craig Whittington, ${ }^{3}$ \\ David Cohen, ${ }^{4}$ Yuqing Zhang, ${ }^{1}$ Peng $X i e^{1}$
}

To cite: Qin B, Zhou X,

Michael KD, et al.

Psychotherapy for depression in children and adolescents: study protocol for a systematic review and network meta-analysis. BMJ Open 2015;5:e005918. doi:10.1136/ bmjopen-2014-005918

- Prepublication history and additional material is available. To view please visit the journal (http://dx.doi.org/ 10.1136/bmjopen-2014005918).

$\mathrm{BQ}$ and $\mathrm{XZ}$ contributed equally.

Received 16 June 2014 Revised 24 October 2014 Accepted 26 November 2014

CrossMark

For numbered affiliations see end of article.

Correspondence to Professor Peng Xie; xiepeng973@126.com

\section{ABSTRACT}

Introduction: Depression is common among children and adolescents and is associated with significantly negative effects. A number of structured psychosocial treatments are administered for depression in children and adolescents; however, evidence of their effectiveness is not clear. We describe the protocol of a systematic review and network meta-analysis to evaluate the efficacy, quality of life, tolerability and acceptability of the use of psychological intervention for this young population.

Methods and analysis: We will search PubMed, EMBASE, CENTRAL (the Cochrane Central Register of Controlled Trials), Web of Science, PsycINFO, CINAHL, LiLACS, Dissertation Abstracts, European Association for Grey Literature Exploitation (EAGLE) and the National Technical Information Service (NTIS) from inception to July 2014. There will be no restrictions on language, publication year or publication type. Only randomised clinical trials (RCTs) with psychosocial treatments for depression in children and adolescents will be considered. The primary outcome of efficacy will be the mean overall change of the total score in continuous depression severity scales from baseline to end point. Data will be independently extracted by two reviewers. Traditional pairwise meta-analyses will be performed for studies that directly compared different treatment arms. Then we will perform a Bayesian network meta-analyses to compare the relative efficacy, quality of life, tolerability and acceptability of different psychological intervention. Subgroup analyses will be performed by the age of participants and the duration of psychotherapy, and sensitivity analyses will be conducted to assess the robustness of the findings.

Ethics and dissemination: No ethical issues are foreseen. The results will be published in a peer-reviewed journal and disseminated electronically and in print. The meta-analysis may be updated to inform and guide management of depression in children and adolescents.

Trials registration number: PROSPERO CRD42014010014.

\section{BACKGROUND}

Depression is increasing rapidly, and ranks fourth on the list of disorders with the highest burden of disease worldwide. ${ }^{1}$ The point
Strengths and limitations of this study

- This Bayesian network meta-analysis can integrate direct evidence with indirect evidence from multiple treatment comparisons to estimate the interrelations across all treatments.

- We will comprehensively assess the efficacy, quality of life, tolerability, acceptability and suiciderelated outcomes in acute treatment and follow-up.

- A series of subgroup and sensitivity analyses will address clinically relevant questions.

- This study should help guide clinical decisionmaking of psychotherapeutic interventions to better treat child and adolescent depression.

prevalence of depression has been estimated to be approximately $2 \%$ in children (6-12 years) and $2-8 \%$ in adolescents (13-18 years), with a peak incidence around puberty. ${ }^{23}$ The average duration of a depressive episode in children and adolescents is about 9 months. ${ }^{4}$ However, $70 \%$ of patients whose symptoms remit develop subsequent depressive episodes within 5 years, and research also shows continuity between childhood depression and depression experienced in adulthood. ${ }^{45}$ Compared with diagnosis of adults, diagnoses of depressed children and adolescents are more often missed $^{5}$ and have more frequent suicide attempts and thoughts, particularly in teenage girls. ${ }^{6}$ There is also serious impairment in social functioning, including poor school achievement and relational problems with family members and peers. ${ }^{78}$

In the past two decades, the availability of effective treatments for depression in children and adolescents has increased, with two broad categories of treatment being used to treat this population: psychotherapy and pharmacotherapy (eg, tricyclic antidepressants (TCAs) and serotonin selective reuptake inhibitors (SSRIs))..$^{9}$ In the management of mild to moderate depression, the first 
treatment option is still psychotherapy no matter which method is used. ${ }^{10}{ }^{11}$ In the USA, approximately three quarters of children and adolescents treated for depression are reported to have received some form of psychotherapy, although approximately one-third of patients underwent only one or two treatment visits during the course of the survey year. ${ }^{12}$ For pharmacotherapy, TCAs are unlikely to benefit children and adolescents with depression, ${ }^{13}$ and treatment with SSRIs has been controversial because of evidence of a significant increase in the risk of suicidality compared to a placebo. ${ }^{14} 15$ Thus, concerns about this issue have refocused attention on the prevalence of depression in young patients, particularly on the most prominent medication alternative, psychotherapy and on the question of how effective this treatment is with youth depression. ${ }^{16}$

The aim of psychotherapy is to build a relationship with the client through a structured and purposeful encounter, and although a range of specific techniques are employed, life issues and problems can be discussed and addressed. Just as there are many approaches to psychological therapies, the assumed mechanism of action for each varies. However, common to most is the aim to increase awareness, with the implicit or explicit aim of changing thoughts, behaviours or emotions to improve the mental health and well-being of the client. ${ }^{17}$ In clinical practice, depressed children and adolescents are more likely to show an irritable mood, rather than the sad mood that features in depressed adults, ${ }^{9}$ and dramatic brain changes have been found between childhood and adult patients in previous studies. ${ }^{18} 19$ Therefore, it is important to analyse psychotherapy use in children and adolescents separately from adults. Since the late 1990s, several rigorous studies have been conducted validating the efficacy of psychotherapeutic approaches in child and adolescent depression. ${ }^{20-22}$ Currently, a number of structured psychosocial treatments are administered for depression in children and adolescents, such as cognitivebehavioural therapy (CBT), which has also been commonly used for adult patients. ${ }^{23}$ Other types of psychotherapies include behavioural, cognitive, interpersonal, problem-solving, play, psychodynamic and family. So far, there have been only a few systematic reviews or meta-analyses for depression in children and adolescents, and the evidence of effectiveness of psychotherapy remains unclear. ${ }^{1624-27}$ One recent meta-analysis by Cox $e t a l^{17}$ found that the existing evidence is not sufficient to robustly determine the relative effectiveness of antidepressants and psychological therapies (alone or in combination) for treating depression in children and adolescents, and another meta-analysis showed a modest benefit from antidepressants with no additional benefit over medication from CBT for treatment-resistant depression in adolescents. ${ }^{26}$ In addition, Hetrick et al $\mathrm{s}^{27}$ study found that the efficacy of psychotherapy varies with different therapeutic components (eg, behavioural therapy may lead to better treatment outcomes in young people). ${ }^{27}$ However, these systematic reviews and meta-analyses have not focused on any head-to-head comparisons of different psychological interventions. Thus, the evidence of hierarchies for the efficacy and tolerability of these interventions in acute phase treatment and follow-up has never been assessed in the comprehensive setting of a systematic review and meta-analysis.

For these reasons, a better-designed approach utilising Bayesian network meta-analysis is urgently needed in this area, integrating direct evidence (from studies directly comparing interventions) with indirect evidence (information about two treatments derived via a common comparator) from multiple treatment comparisons to estimate the interrelations across all treatments. ${ }^{28}$ This approach enables a coherent analysis of random trials data for comparisons of multiple treatments without adversely affecting randomisation of treatments within each trial, and its usefulness has been previously demonstrated in several studies on various medical conditions and interventions, such as antidepressants or psychotherapy for depression in adults, ${ }^{29} 30$ and antimanic drugs for mania in adults. ${ }^{31}$ Recently, we have completed a network meta-analysis comparing first-generation and secondgeneration antidepressants for depression in children, adolescents and young adults. To further improve the management for depression in children and adolescents, we will conduct a systematic review and network metaanalysis to evaluate the efficacy, quality of life, tolerability and acceptability of the use of psychological intervention for this young population.

\section{METHODS \\ Criteria for included studies \\ Types of studies}

Any relevant randomised controlled trials (RCTs) will be included. Quasi-randomised trials (such as those allocating by using alternate days of the week) will be excluded. For trials that have a crossover design, only the results from the first randomisation period will be considered. Also, the cluster RCTs will be included.

\section{Types of participants}

Children or adolescents should be between the ages of 6 and 18 years when they initially participated in the primary studies. For the purpose of reducing clinical heterogeneity, investigators always use one strict diagnostic definition, such as Diagnostic and Statistical Manual of Mental Disorders, fourth edition (DSM-IV) or International Classification of Diseases 10th revision (ICD-10), to diagnose major depression. However, it is most likely that this method could overlook a lot of important information from clinical settings, because some children and adolescents, who do not satisfy the typical criteria of major depression but suffer from depressive symptoms or subthreshold depression, can also have serious functional deterioration in social and educational situations. Therefore, the following broad criteria to identify the participants will be applied in our 
study:(1) major depression as diagnosed according to standardised criteria such as DSM-III, ${ }^{32}$ DSM-III-R, ${ }^{33}$ DSM-IV $^{34}$ ICD-9, ${ }^{35}$ ICD-10, ${ }^{36}$ Research Diagnostic Criteria $^{37}$ and Feighner criteria, ${ }^{38}$ (2) minor or intermittent depression, or dysthymia as diagnosed according to standardised criteria such as DSM-III, DSM-III-R, DSM-IV, ICD-9, ICD-10, Research Diagnostic Criteria and Feighner criteria, (3) depressive status, defined as scoring above a certain cut-off on a depression rating scale according to the original authors' definition, such as the Hamilton Rating Scale for Depression (HRSD), ${ }^{39}$ Children's Depression Inventory (CDI), ${ }^{40}$ Beck depression inventory (BDI), ${ }^{41}$ Center for Epidemiologic Study Depression Scale(CES-D), ${ }^{42}$ Children's Depression Rating Scale (CDRS) ${ }^{43}$ or Children's Depression Rating Scale-Revised (CDRS-R). ${ }^{44}$ Trials where adults and children or adolescents are treated will be eligible for inclusion, if data on the children or adolescents could be extracted separately or obtained from trial authors. The studies where participants had comorbid secondary medical or other mental health conditions, including comorbid with suicidal ideation/attempt will not be excluded; however, participants with a secondary diagnosis of Axis I psychiatric disorders (eg, schizophrenia and bipolar disorder) will be excluded because the effectiveness of psychotherapy might be affected by these comorbidities. Besides, studies in which participants have a diagnosis of resistant depression or psychotic depression will be excluded.

\section{Types of interventions}

RCTs comparing one psychological intervention with another or either of the control conditions for depression in children and adolescents will be included. For psychotherapy, CBT, behavioural therapy (BT), cognitive therapy (CT), interpersonal therapy, problem-solving therapy, play therapy, supportive counselling, psychodynamic therapy and family therapy will be included regardless of their treatment session and duration. The psychotherapy will be shown in table 1 . We will view these psychological interventions as independent nodes in this network meta-analysis regardless of the treatment medium (face-to-face, website, telephone or other). If sufficient numbers of studies compared between different treatments in the same type of psychotherapy as currently described are detected, we will consider viewing it as another new node because it seems that the effect of each type of psychotherapy is unique.

In terms of control conditions, waiting-list control (WL), non-treatment control, treatment as usual (TAU) and (psychological or pill) placebo will be included. TAU is not considered to be psychotherapy but may have some treatment effects. Placebo may also have some potential treatment effects, whereas non-treatment and $\mathrm{WL}$ do not have any active treatment effects. Therefore, we will also view these control conditions as independent nodes in this network meta-analysis, except that the non-treatment and waiting list will be regarded as one node. Regular reviews of mental health symptoms and interventions, such as psychodrama and art exercise, will be regarded as psychological placebos because they involve time spent with the patient but without a focus on improving depressive symptoms. ${ }^{24}$

Trials in which psychotherapy is used as a combination strategy (such as combining different psychological interventions or psychotherapy and antidepressants) will be excluded, while studies with concomitant use of an auxiliary psychotropic agent (eg, benzodiazepine for insomnia) will be included.

\section{Types of outcome measures}

\section{Acute phase treatment}

We will assess the effects of acute phase treatment posttreatment in order to examine the possible maximum effects of psychotherapy.

\section{Overall efficacy}

1. The primary outcome of efficacy will mean overall change in the total score in continuous depression severity scales from baseline to end point, which will be assessed in the first instance by change in HRDS. If data are not available, we will use change in BDI or CDI and then other depressive rating scales.

2. Secondary outcome of efficacy will be the proportion of patients who respond to treatment, which is defined as substantial overall improvement from baseline as defined by the original investigators, such as more than a $50 \%$ reduction on a depression continuous measure (ie, ' $1=$ =ery much improved' or '2=Much improved' according to the Clinical Global Improvement (CGI) Scale or other criteria). ${ }^{42}$ When 'response' is not reported, we will use 'remission' if available. Remission is defined as a reduction to the normal range (such as HRSD score $\leq 9$, BDI score $\leq 10, \mathrm{CDI} \leq 12)$ and variably across studies. ${ }^{45}$

\section{Overall acceptability}

All-cause discontinuation as a proxy measure of treatment acceptability is defined as the proportion of patients who drop out for any reason.

\section{Overall tolerability}

Side effects discontinuation as a measure of treatment tolerability is defined as the proportion of patients who drop out for any reason.

\section{Quality of life/functioning improvement}

Quality of life/functioning improvement (QoL/functioning) means overall change in the total score in continuous quality of life scales from baseline to end point, for example, Quality of Life Enjoyment and Satisfaction Questionnaire (Q-LES-Q) ${ }^{46}$ and the Short Form 36 Health Survey (SF-36), ${ }^{47}$ etc, or functioning improvement scales, for example, Sheehan Disability Scale (SDS). ${ }^{48}$ As measures of QoL/functioning vary across 
Table 1 Description of intervention strategies

\begin{tabular}{|c|c|c|}
\hline $\begin{array}{l}\text { Psychological } \\
\text { intervention }\end{array}$ & Abbreviation & Description \\
\hline $\begin{array}{l}\text { Cognitive behavioural } \\
\text { therapy }\end{array}$ & CBT & $\begin{array}{l}\text { CBT uses some kind of cognitive restructuring training and promotes behavioural } \\
\text { change }\end{array}$ \\
\hline Behavioural therapy & BT & $\begin{array}{l}\text { It uses some kind of behavioural training and thus promotes cognitive change. It } \\
\text { may include relaxation therapy, biofeedback, coping skills, behavioural activation } \\
\text { or social skills training }\end{array}$ \\
\hline Cognitive therapy & CT & $\begin{array}{l}\text { It uses some kind of cognitive restructuring training but does not promote } \\
\text { behavioural change }\end{array}$ \\
\hline Interpersonal therapy & IPT & $\begin{array}{l}\text { IPT is a brief and highly-structured manual-based psychotherapy that focuses on } \\
\text { the participants' social relationships and current evaluation of these relationships }\end{array}$ \\
\hline Problem-solving therapy & PST & $\begin{array}{l}\text { PST focuses on the problems participants currently face and on helping them find } \\
\text { solutions to these problems }\end{array}$ \\
\hline Play therapy & - & $\begin{array}{l}\text { It uses techniques to engage participants in recreational activities to help them } \\
\text { cope with their problems and fears }\end{array}$ \\
\hline $\begin{array}{l}\text { Non-directive supportive } \\
\text { therapy }\end{array}$ & SUP & $\begin{array}{l}\text { SUP is an unstructured therapy without specific psychological techniques that } \\
\text { helps people to express their experiences and emotions and offer empathy. } \\
\text { These nondirective therapies are commonly described in the literature as either } \\
\text { counselling or supportive therapy }\end{array}$ \\
\hline Psychodynamic therapy & DYN & $\begin{array}{l}\text { DYN refers to a technique designed to help a person understand the origin and } \\
\text { nature of long-standing problems, including psychological trauma }\end{array}$ \\
\hline Family therapy & - & $\begin{array}{l}\text { It works with families and couples in intimate relationships to nurture change and } \\
\text { development. It tends to view change in terms of the systems of interaction } \\
\text { between family members }\end{array}$ \\
\hline
\end{tabular}

studies, we will pool such measures together to create an omnibus effect size for each psychotherapy.

\section{Suicide-related outcomes}

We consider suicide-related outcomes as both a dichotomous and continuous outcome. If data are available, we will extract the number of participants with suicidal behaviour/ideation during the acute treatment, as measured on a standardised, validated and reliable rating scale such as the Suicidal Ideation Questionnaire-Junior High School version. ${ }^{49}$ In addition, we will also collect data on suicidal ideation as a continuous outcome where a standardised, validated and reliable rating scale has been used.

\section{Follow-up measure}

After the acute phase treatment, we will assess the effects at the end of follow-up (maximum of up to 12 months) in order to examine the possible delayed or maintenance effects of psychotherapy. If some participants took further treatments, including continuous treatment, booster sessions or any other psychotherapies or antidepressants during the follow-up period, we will exclude them in the follow-up analysis. Therefore, the number of participants who did not take further treatments will be used as a measure of follow-up efficacy of the acute phase interventions. Owing to the variability in follow-up points, and the fact that some studies assessed outcomes at multiple follow-up time points, we will collect data at the last time points as an outcome measure.

\section{Overall efficacy}

1. The primary outcome of efficacy in the long term will be defined as the mean overall change in continuous depression severity scales, which is similar to the acute phase treatment measurement.

2. The secondary outcome of efficacy will be defined as 'response', which is similar to the acute phase treatment measurement.

\section{Overall acceptability}

All-cause discontinuation as a proxy measure of treatment acceptability is defined as the proportion of patients who drop out for any reason.

\section{Overall tolerability}

Side effects discontinuation as a measure of treatment tolerability is defined as the proportion of patients who drop out for any reason.

\section{Quality of life/functioning improvement}

QoL/functioning will be the mean overall change in continuous quality of life scales or functioning improvement scales, which is similar to the acute phase treatment measurement.

\section{Suicide-related outcomes}

We consider suicide-related outcomes as both a dichotomous and continuous outcome. If data are available, we will extract the number of participants with suicidal behaviour/ideation during the acute treatment, as measured on a standardised, validated and reliable rating 
scale such as the Suicidal Ideation Questionnaire-Junior High School version. ${ }^{49}$ In addition, we will also collect data on suicidal ideation as a continuous outcome where a standardised, validated and reliable rating scale has been used.

\section{Search strategy}

All published, unpublished and ongoing RCTs that compared one psychological intervention with another or either of the control conditions in the treatment of depression in children and adolescents will be identified. We will identify relevant trials from systematic searches in the following electronic databases: MEDLINE/PubMed, EMBASE, CENTRAL (the Cochrane Central Register of Controlled Trials), Web of Science, PsycINFO, CINAHL and LiLACS. A comprehensive search of unpublished theses and dissertations via ProQuest Dissertation Abstracts, European Association for Grey Literature Exploitation (EAGLE) and National Technical Information Service (NTIS) will be completed. We will search clinicaltrials.gov for ongoing trial registers. We will also check relevant reports on the US Food and Drug Administration (FDA) website, the WHO's trial portal, and hand-search major psychiatric and medical journals. The search deadline will be from inception up to July 2013. No language restrictions will be applied. Details of search strategies can be found in online supplementary appendix table 1. Electronic databases will be searched using the following strategy: additional relevant studies will be obtained by scanning reference lists of trials identified in the initial searches and relevant review papers. In addition, all relevant authors will be contacted to supplement incomplete information.

\section{Study selection and data extraction \\ Selection of trials}

Titles and abstracts of references identified by the electronic search strategies described above will be independently examined by two reviewers (BQ and YL). If both reviewers judge that the trial does not meet eligibility criteria, we will exclude it. Then we will obtain the full texts of all remaining articles and determine whether to include them by the same eligibility criteria. The inter-rater reliability of the two raters will be calculated. Besides, the references of relevant review papers and included trials will be checked by $\mathrm{BQ}$ and $\mathrm{YZ}$. Any disagreement will be resolved by consensus between the two reviewers and, if need be, with another reviewer (XZ). The reasons for exclusion of trials will be reported in the characteristics of excluded studies tables.

\section{Quality assessment}

Two reviewers (BQ and YL) will independently assess the methodological quality of the included studies. We will assess risk of bias as 'low risk', 'unclear risk' or 'high risk', in accordance with The Cochrane Collaboration's ' Risk of bias' tool as described in the Cochrane
Handbook for Systematic Reviews of Interventions. ${ }^{50}$ The following items will be assessed:

1. Random sequence generation.

2. Allocation concealment.

3. Blinding of participants, personnel and outcome assessors (assessments should be made for each main outcome or class of outcomes).

4. Incomplete outcome data (assessments should be made for each main outcome or class of outcomes).

5. Selective outcome reporting.

6. Other sources of bias.

The inter-rater reliability of the total score of study quality assessment will be examined. Any disagreement will be resolved by consensus between the two raters and, if need be, with another reviewer (XZ).

\section{Data extraction}

Two independent reviewers (BQ and YL) will extract the data from the original reports using standardised data extraction forms, which include $f$ study characteristics (such as first listed author, publication year, journal, country, institution and sponsor), patient characteristics (such as diagnostic criteria for depression, the type of patients, the number of patients and patients' baseline), intervention details (such as psychotherapy type, session of psychotherapy, the duration of treatment, treatment setting and pattern) and outcome measures (such as the patients of intention-to-treat (ITT) analysis, acute treatment outcomes and follow-up outcomes). Any disagreements will be resolved by a third review author (XZ).

\section{Statistical analysis}

First, traditional pairwise meta-analyses will be performed for studies that directly compared different treatment arms. Then we will perform a Bayesian network meta-analysis to assess the relative outcomes of different psychotherapies and control conditions with each other from all direct and indirect comparisons. Dichotomous outcomes will be analysed on an ITT basis: dropouts will always be included in this analysis. When data on dropouts are carried forward and included in the evaluation (Last Observation Carried Forward, LOCF), they will be analysed according to the primary studies. Scores from continuous outcomes will not be analysed on an ITT basis. Continuous outcomes will therefore be analysed on an end point basis, including only participants with a final assessment or with a LOCF to the final assessment.

\section{Traditional pairwise meta-analyses}

Traditional pairwise meta-analyses will be performed using Review Manager (V.5.2). Using the DerSimonian method and the Laird random-effects model, the pooled estimates of standardised mean difference (SMD) with 95\% CIs will be calculated for the continuous outcomes, and ORs with 95\% CIs will be calculated for the dichotomous outcomes. Heterogeneity of treatment effects across studies will be assessed by $\mathrm{I}^{2}$ and the Q-statistic test. ${ }^{50}$ 


\section{Bayesian network meta-analyses}

Network meta-analyses will be performed using the WinBUGS software package (V.1.4.3, MRC Biostatistics Unit, Cambridge, UK) with random-effects models for multiarm trials. ${ }^{28} 51$ The other analyses will be performed and presented by the Stata V.11.0 and R V.2.11.1 software packages. Network meta-analyses will be performed on two different evidence networks. The primary analysis is based on a network where the psychological interventions mentioned above will be treated as a separate node regardless of group or individual treatment. A secondary evidence network of drug class will also be constructed to view the group psychotherapy or individual psychotherapy in the same intervention as a separate node. The pooled estimates will be obtained using the Markov Chains Monte Carlo method. Two Markov chains will be run simultaneously with different arbitrarily chosen initial values. To ensure convergence, trace plots and the Brooks-GelmanRubin statistic will be assessed. ${ }^{52}$ Convergence will be found to be adequate after running 50000 samples for both chains. These samples will then be discarded as 'burn-in,' and posterior summaries will be based on 100000 subsequent simulations. All results will be reported as posterior medians of SMD or with corresponding $95 \%$ credible intervals (CrIs), which can be interpreted like conventional 95\% CIs. When a loop connected three treatments, it was possible to evaluate the inconsistency between direct and indirect evidence. The node splitting method will be used to calculate the inconsistency of the model, which separates evidence on a particular comparison into direct and indirect evidence. ${ }^{53}$

The probability of each treatment being the most effective (best, second-best, third-best and so on) will be calculated and graphically ranked with rankograms. ${ }^{54}$ Probability values will be summarised and reported as surface under the cumulative ranking curve (SUCRA), a simple transformation of the mean rank used to provide a hierarchy of the treatments that accounts for the location and the variance of all relative treatment effects. ${ }^{55}$ The larger the SUCRA value, the better the rank of the treatment with a SUCRA of 1.0 if an intervention always ranks first and 0.0 if it always ranks last.

\section{Subgroup analysis}

Where possible, we will perform the following subgroups analysis: (1) for the age of participants (eg, children aged 6-12 years or adolescents from 13 to 18 years); and (2) for the duration of psychotherapy (eg, short-term treatment of six or fewer weeks or long-term treatment of more than 6 weeks).

\section{Sensitivity analyses}

We will perform the following sensitivity analyses: (1) by limiting the studies to be included to those of higher quality; (2) by limiting studies to those that employed standardised criteria for major depression; (3) by excluding studies with comorbid physical or mental disorders that were not a primary diagnosis (if possible, excluding only participants who had a comorbid diagnosis); (4) by excluding studies with a concomitant use of psychotropic agents (if possible, excluding only participants who had a concomitant use of psychotropic agents); (5) by excluding studies with small sample sized trials; and (6) by excluding studies with mild and intermittent depression patients.

\section{Funnel plot analysis and meta-regression analysis}

We will perform funnel plot analyses to check for publication bias. Moreover, we will carry out meta-regression analyses to investigate the effect of sponsorship or year published on outcome estimate.

\section{Ethics and dissemination}

This systematic review and network meta-analysis will be published in a peer-reviewed journal. It will be disseminated electronically and in print. As no primary data collection will be undertaken, no additional formal ethical assessment and informed consent are required.

\section{Author affiliations}

${ }^{1}$ Department of Neurology, The First Affiliated Hospital of Chongqing Medical University, Chongqing, China

${ }^{2}$ Department of Psychology, Appalachian State University, Boone, North Carolina, USA

${ }^{3}$ Centre for Outcomes Research and Effectiveness, Research Department of Clinical, Educational \& Health Psychology, University College London, London, UK

${ }^{4}$ Sorbonne Universités; and the Department of Child and Adolescent Psychiatry, Institut des Systèmes Intelligents et de Robotiques, UPMC Univ Paris 06, UMR 7222, AP-HP, Hôpital Pitié-Salpétrière, Paris, France

Acknowledgements The authors thank the scientific editors at Impactys (http://www.impactys.com) for editing and proofreading this manuscript.

Contributors $B Q$ and $X Z$ conceived the study and drafted the protocol. KDM, $\mathrm{CW}$ and $\mathrm{DC}$ assisted in protocol design. $\mathrm{YL}$ and $\mathrm{YZ}$ have participated in the search strategy development. All authors read and approved the final version of the manuscript.

Funding This work was supported by the National Basic Research Program of China (973 Program) (Grant No. 2009CB918300) and University College London's own open access fund.

\section{Competing interests None.}

Provenance and peer review Not commissioned; externally peer reviewed.

Open Access This is an Open Access article distributed in accordance with the Creative Commons Attribution Non Commercial (CC BY-NC 4.0) license, which permits others to distribute, remix, adapt, build upon this work noncommercially, and license their derivative works on different terms, provided the original work is properly cited and the use is non-commercial. See: http:// creativecommons.org/licenses/by-nc/4.0/

\section{REFERENCES}

1. The World Health Organization. Annex Table 3 Burden of disease in $D A L Y$ s by cause, sex and mortality stratum in WHO Regions, a estimates for 2002. The World Health Report, 2004.

2. Costello EJ, Mustillo S, Erkanli A, et al. Prevalence and development of psychiatric disorders in childhood and adolescence. Arch Gen Psychiatry 2003;60:837-44.

3. Birmaher B, Ryan ND, Williamson DE, et al. Childhood and adolescent depression: a review of the past 10 years, part I. J Am Acad Child Adolesc Psychiatry 1996;35:1427-39.

4. Philip H. Depression in children may go unnoticed and untreated. BMJ 2002;325:229-30. 
5. Thapar A, Collishaw S, Pine DS, et al. Depression in adolescence. Lancet 2012;379:1056-67.

6. Dodig-Curković K, Curković M, Radić J, et al. Suicidal behavior and suicide among children and adolescents-risk factors and epidemiological characteristics. Coll Antropol 2010;34:771-7.

7. Puig-Antich J, Lukens $\mathrm{E}$, Davies $\mathrm{M}$, et al. Psychosocial functioning in prepubertal major depressive disorders. II. Interpersonal relationships after sustained recovery from affective episode. Arch Gen Psychiatry 1985;42:511-17.

8. Jaffee SR, Moffitt TE, Caspi A, et al. Differences in early childhood risk factors for juvenile-onset and adult-onset depression. Arch Gen Psychiatry 2002;59:215-22.

9. National Collaborating Centre for Mental Health (UK). Depression in Children and Young People: Identification and management in primary, community and secondary care. British Psychological Society, 2005. http://www.ncbi.nlm.nih.gov/books/NBK56425/ (accessed 28 Nov 2005)

10. The Chief Executive Officer of the National Health and Medical Research Council (NHMRC). Clinical Practice Guidelines: Depression in Adolescents and Young Adults. https://www.bspg. com.au/dam/bsg/product?client=BEYONDBLUE\&prodid=BL 0890\&type=file (accessed 11 Feb 2011).

11. Cheung $A H$, Zuckerbrot RA, Jensen PS, et al. Guidelines for Adolescent Depression in Primary Care (GLAD-PC): II. Treatment and ongoing management. Pediatrics 2007;120:e1313-26.

12. Olfson M, Gameroff MJ, Marcus SC, et al. Outpatient treatment of child and adolescent depression in the United States. Arch Gen Psychiatry 2003;60:1236-42.

13. Hazell $P$, Mirzaie $M$. Tricyclic drugs for depression in children and adolescents. Cochrane Database Syst Rev 2013;6:CD002317.

14. U.S. Food and Drug Administration (FDA). Suicidality in children and adolescents being treated with antidepressant medications. http:// www.fda.gov/cder/drug/antidepressants/SSRI-PHA200410.htm (accessed 11 Oct 2004)

15. Hetrick S, McKenzie J, Cox R, et al. Newer generation antidepressants for depressive disorders in children and adolescents. Cochrane Database Syst Rev 2012;11:CD004851.

16. Weisz JR, McCarty CA, Valeri SM. Effects of psychotherapy for depression in children and adolescents: a meta-analysis. Psychol Bull 2006;132:132-49.

17. Cox GR, Callahan P, Churchill R, et al. Psychological therapies versus antidepressant medication, alone and in combination for depression in children and adolescents. Cochrane Database Syst Rev 2012;11:CD008324.

18. Kaufman J, Martin A, King RA, et al. Are child-, adolescent-, and adult-onset depression one and the same disorder? Biol Psychiatry 2001;49:980-1001.

19. Blakemore SJ, Choudhury S. Development of the adolescent brain: implications for executive function and social cognition. J Child Psychol Psychiatry 2006:47:296-312.

20. Mufson L, Dorta KP, Wickramaratane $\mathrm{P}$, et al. A randomized effectiveness trial of interpersonal psychotherapy for depressed adolescents. Arch Gen Psychiatry 2004;61:577-84.

21. Brent DA, Holder D, Kolko D, et al. A clinical psychotherapy trial for adolescent depression comparing cognitive, family and supportive treatments. Arch Gen Psychiatry 1997;54:877-85.

22. Muratori F, Picchi L, Bruni G, et al. A two-year follow-up of psychodynamic psychotherapy for internalizing disorders in children. J Am Acad Child Adolesc Psychiatry 2003;42:331-9.

23. Cuijpers $P$, van Straten A, Andersson G, et al. Psychotherapy for depression in adults: a meta-analysis of comparative outcome studies. J Consult Clin Psychol 2008;76:909-22.

24. Watanabe N, Hunot V, Omori IM, et al. Psychotherapy for depression among children and adolescents: a systematic review. Acta Psychiatr Scand 2007;116:84-95.

25. David-Ferdon C, Kaslow NJ. Evidence-based psychosocial treatments for child and adolescent depression. J Clin Child Adolesc Psychol 2008;37:62-104.

26. Hetrick SE, Cox GR, Merry SN. Treatment-resistant depression in adolescents: is the addition of cognitive behavioral therapy of benefit? Psychol Res Behav Manag 2011;4:97-112.

27. Hetrick SE, Cox GR, Fisher CA, et al. Back to basics: could behavioural therapy be a good treatment option for youth depression? A critical review. Early Interv Psychiatry 2014. doi:10.1111/eip.12142
28. Salanti G, Higgins JP, Ades AE, et al. Evaluation of networks of randomized trials. Stat Methods Med Res 2008;17:279-301.

29. Cipriani A, Furukawa TA, Salanti G, et al. Comparative efficacy and acceptability of 12 new-generation antidepressants: a multipletreatments meta-analysis. Lancet 2009;373:746-58.

30. Barth J, Munder T, Gerger H, et al. Comparative efficacy of seven psychotherapeutic interventions for patients with depression: a network meta-analysis. PLoS Med 2013;10:e1001454.

31. Cipriani A, Barbui C, Salanti G, et al. Comparative efficacy and acceptability of antimanic drugs in acute mania: a multipletreatments meta-analysis. Lancet 2011;378:1306-15.

32. American Psychiatric Association (APA). Diagnostic and statistical manual of mental disorders (DSM-III). 3rd edn. Washington DC: American Psychiatric Association, 1980.

33. American Psychiatric Association (APA). Diagnostic and statistical manual of mental disorders (DSM-III-R). 3rd revised edn. Washington DC: American Psychiatric Association, 1987.

34. American Psychiatric Association (APA). Diagnostic and statistical manual of mental disorders. 4th edn. Washington DC: American Psychiatric Association, 1994.

35. World Health Organization (WHO). The ninth revision of the international classification of diseases and related health problems (ICD-9). Geneva: World Health Organization, 1978.

36. World Health Organization (WHO). The tenth revision of the international classification of diseases and related health problems (ICD-10). Geneva: World Health Organization, 1992.

37. Spitzer RL, Endicott J, Robins E. Research diagnostic criteria. Psychopharmacol Bull 1975;11:22-5.

38. Feighner JP, Robins E, Guze SB, et al. Diagnostic criteria for use in psychiatric research. Arch Gen Psychiatry 1972;26: 57-63.

39. Hamilton M. A rating scale for depression. J Neurol Neurosurg Psychiatry 1960;23:56-62.

40. Kovacs M. The Children's Depression, Inventory (CDI). Psychopharmacol Bull 1985;21:995-8.

41. Beck AT, Steer R. Beck depression inventory: manual. San Antonio, TX: Psychological Corporation, 1987.

42. Radloff LS. The CES-D scale: a self-report depression scale for research in the general population. Appl Psychol Meas 1977:1:385-401.

43. Poznanski EO, Cook SC, Carroll BJ. A depression rating scale for children. Pediatrics 1979;64:442-50.

44. Posnanski EO, Freeman LN, Mokros HB. Children's depression rating scale revised. Psychopharmacol Bull 1984;21:979-89.

45. Riedel M, Moller HJ, Obermeier M, et al. Response and remission criteria in major depression - a validation of current practice. J Psychiatr Res 2010;44:1063-8.

46. Endicott J, Nee J, Harrison W, et al. Quality of life enjoyment and satisfaction questionnaire: a new measure. Psychopharmacol Bull 1993;29:321-6.

47. Ware J, Sherbourne CD. The MOS 36-item short-form health survey (SF-36). Med Care 1992;30:473-83.

48. Sheehan D, Harnett-Sheehan K, Raj B. The measurement of disability. Int Clin Psychopharmacol 1996;11(Suppl 3):89-95.

49. Reynolds WM. Suicidal ideation questionnaire-junior. Odessa, FL: Psychological Assessment Resources, 1987.

50. JPT CHH, Green S. Cochrane handbook for systematic reviews of interventions version 5.1. O [updated March 2011]. The Cochrane Collaboration, 2011. http://www.cochrane-handbook.org (accessed 1 Oct 2011)

51. Lu G, Ades AE. Combination of direct and indirect evidence in mixed treatment comparisons. Stat Med 2004;23:3105-24.

52. Brooks SP, Gelman A. Alternative methods for monitoring convergence of iterative simulations. J Comput Graph Stat 1998;7:434-45.

53. Lu G, Ades A. Assessing evidence inconsistency in mixed treatment comparisons. J Am Stat Assoc 2006;101:447-59.

54. Salanti G, Ades AE, loannidis JP. Graphical methods and numerical summaries for presenting results from multiple-treatment meta-analysis: an overview and tutorial. J Clin Epidemiol 2011;64:163-71.

55. van der Valk R, Webers CA, Lumley $\mathrm{T}$, et al. A network meta-analysis combined direct and indirect comparisons between glaucoma drugs to rank effectiveness in lowering intraocular pressure. J Clin Epidemiol 2009;62:12. 